\title{
Photometric binary content in intermediate/old open clusters
}

\author{
T. S. Sandhu, A. K. Pandey, and R. Sagar
}

\author{
State Observatory, Manora Peak, Naini Tal, 263129 Uttaranchal, India
}

Received 13 September 2002 / Accepted 4 June 2003

\begin{abstract}
The stellar evolutionary models by Bertelli et al. (1994) have been used to generate synthetic colour-magnitude diagrams (CMDs) of open clusters. A comparison of the synthetic integrated luminosity function (ILF) and synthetic colour distribution with the corresponding observed distributions is used to estimate the photometric binary content in three intermediate age open clusters, which is found to be about $30 \%$ in these clusters.
\end{abstract}

Key words. open clusters and associations: general - stars: Hertzsprung Russell (HR) and C-M diagrams

\section{Introduction}

The mass function (MF), defined as the distribution of stars as a function of mass in a defined volume, is an important parameter that constrains theoretical studies concerning the star formation processes. The MF of open cluster is usually obtained from the observed luminosity function (LF), which can be obtained from the observed colour-magnitude diagrams (CMDs). The CMDs of open clusters usually show a well-defined long and broad main sequence (MS) (see e.g. Durgapal et al. 2001). The breadth of the MS may be due to various reasons, e.g., photometric errors, presence of unresolved binaries, presence of field stars and variable reddening. In the case of intermediate and old open clusters (age $>300 \mathrm{Myr}$ ) the reddening is generally uniform (Pandey et al. 1990; Yadav \& Sagar 2001) across the cluster face. Therefore photometric errors and presence of unresolved binaries are the only main reasons that cause broadening of the MS, if the field star contamination is accounted for.

The MF of intermediate/old open clusters has been studied by several authors (e.g. Aparicio et al. 1990; Kaluzny \& Rucinski 1995; Sagar \& Griffiths 1998; Durgapal \& Pandey 2001 and references therein). In most of the studies, the mass of a star was inferred from the observed luminosity, which may not be correct if the star is an unresolved binary. In the literature, evidence for the presence of binaries in open clusters exists. For example, Mermilliod \& Mayor (1989) found $25 \%-33 \%$ spectroscopic binaries in open clusters. Aparicio et al. (1990) and Durgapal et al. (2001) reported $>25 \%$ and $10 \%-20 \%$ photometric unresolved binaries in the clusters. In the case of the Pleiades cluster, Bouvier et al. (1997) reported a binary (wider,visual) frequency of about $28 \pm 4 \%$ for $\mathrm{G}$ and $\mathrm{K}$ dwarfs. Using the infrared speckle observations of the Hyades cluster, Patience et al. (1998) found that $40 \%$

Send offprint requests to: A. K. Pandey,

e-mail: pandey@upso.ernet.in stars are binary. Recently, Jeffries et al. (2001), for the cluster NGC 2516, found a photometric binary fraction of $26 \pm 5 \%$ for A to M-type systems with mass ratio between 0.6 and 1 . Therefore in the presence of photometric binaries in open clusters, the conversion of observed LF will not yield a true MF.

Over the last decade synthetic CMDs have been used to study various properties of the clusters, e.g. the MF and the influence of unresolved binaries on the luminosity function etc. (e.g. Chiosi et al. 1989; Aparicio et al. 1990; Sagar \& Richtler 1991; Sagar \& Subramaniam 1995; Siess et al. 1997; Brocato et al. 1999). In the present study we make an attempt to estimate the photometric binary contribution in open clusters and their effect on the MF, using the synthetic CMDs of the clusters. In Sect. 2 we describe our approach to derive the synthetic CMDs in the presence of binaries. In Sect. 3 we attempt to estimate the percentage of photometric binaries in open clusters using their CMDs.

\section{Synthetic colour-magnitude diagram}

The synthetic CMDs are constructed using the technique described by Chiosi et al. (1989) and Subramaniam \& Sagar (1995). Briefly, this technique consists of random generation of stars by means of a Monte Carlo technique and distributing the stars along a given isochrone according to evolutionary phases and the initial mass function (IMF). The following expression is used to describe the IMF,

$\mathrm{d} N=A M^{-x} \mathrm{~d} M$

where $\mathrm{d} N$ is the number of stars in the mass interval $\mathrm{d} M, x$ is the slope of the mass function. The Salpeter (1955) value for the slope is 2.35 (1.35 if mass interval is logarithmic). The constant $A$ is fixed by the number of stars present in the brightest portion of the observed MS. 
Table 1. The details of the clusters used in the study.

\begin{tabular}{lrrlllll}
\hline \hline Cluster & $l$ & $b$ & $\begin{array}{l}E(B-V) \\
(\mathrm{mag})\end{array}$ & $\begin{array}{l}(m-M)_{V} \\
(\mathrm{mag})\end{array}$ & $\begin{array}{l}\text { Log age } \\
(\mathrm{Yr})\end{array}$ & $\begin{array}{l}\text { Mass range } \\
\left(M_{\odot}\right)\end{array}$ & References \\
\hline King 5 & 143.7 & -4.3 & 0.82 & 13.95 & 9.0 & $0.9-1.8$ & Durgapal et al. (2001) \\
King 7 & 149.7 & 1.0 & 1.25 & 15.6 & 8.8 & $1.1-2.4$ & Durgapal et al. (1997) \\
M 37 & 177.7 & 3.1 & 0.36 & 11.6 & 8.6 & $0.6-2.4$ & Nilakshi \& Sagar (2002) \\
\hline
\end{tabular}

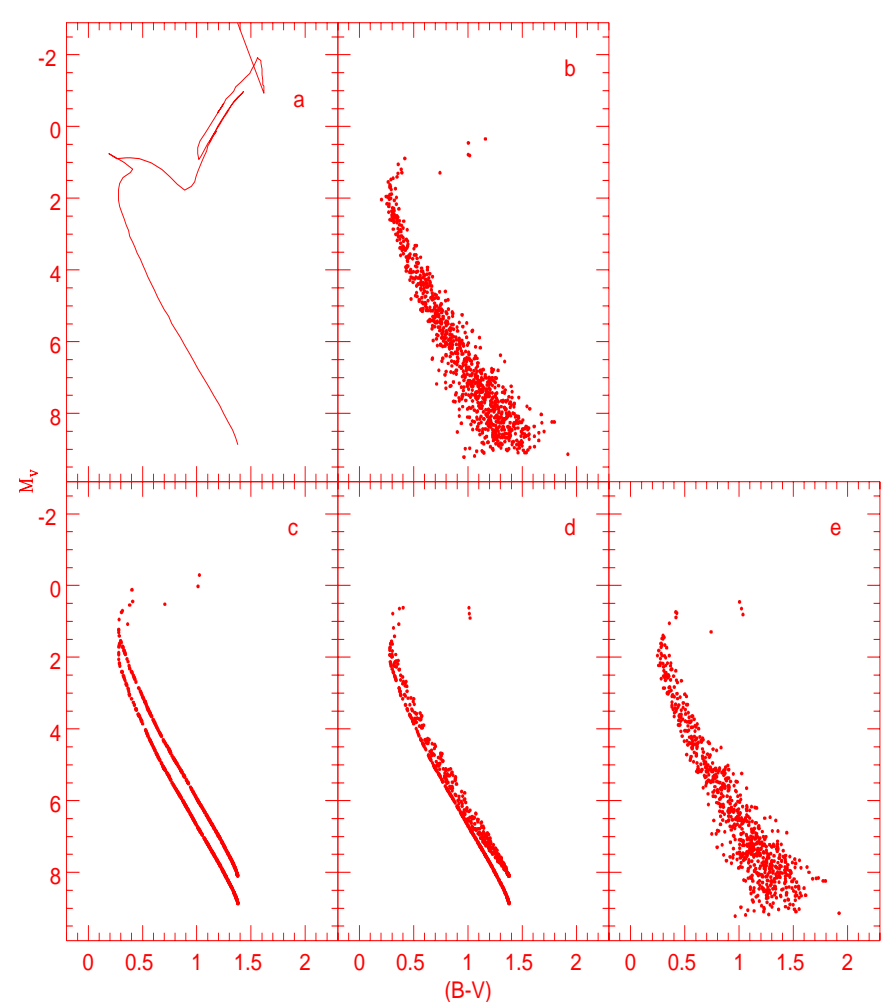

Fig. 1. Construction of synthetic colour-magnitude diagram. a) Isochrones by Bertelli et al. (1994) for $0.4 \mathrm{Gyr}$ age and solar metallicity. b) Synthetic CMD for a cluster having age $0.4 \mathrm{Gyr}$ and $Z=0.02$. The stars are distributed as per the Salpeter mass function with slope $x=2.35$. c) Synthetic CMD for a cluster having $30 \%$ equal mass binary content. The photometric errors are switched off. d) Same as Fig. 1c but for mass ratio of binary content that varies between 0.75 and 1.0. e) Final synthetic CMD which includes assumed binary content and photometric errors. For details see text.

The simulations have been carried out using the evolutionary models with convective overshooting given by Bertelli et al. (1994). Figure 1a shows an isochrone by Bertelli et al. (1994) for age $=0.4 \mathrm{Gyr}$ and $Z=0.02$. In an ideal condition, stars of a cluster having age 0.4 Gyr should lie on the isochrone. As discussed earlier, observational uncertainties introduce dispersion in the magnitude and colours of the stars, consequently we see dispersion in the features of the observed CMDs. The observational uncertainties are introduced assuming a Gaussian distribution for the errors having the standard deviation $\delta V=a(V-b)^{2}$ and $\delta B=c(B-d)^{2}$. The coefficients $a, b, c$ and $d$ are selected to fit the observational trend of the errors as a function of brightness. Figure $1 \mathrm{~b}$ shows a synthetic CMD for a cluster having age $0.4 \mathrm{Gyr}$ and $Z=0.02$. The stars are distributed as per the Salpeter law having $x=$ 2.35. As can be seen, the synthetic CMDs reproduce the features of the observed CMDs.

Another factor that contributes to spread the MS band is the presence of photometric binaries. The presence of photometric binaries among MS stars cannot be disregarded as at any given colour MS stars may be brightened by $0.75 \mathrm{mag}$, if both components of the photometric binaries have the same mass, and consequently can alter the LF. In order to evaluate the effect of photometric binary stars on the shape of the CMDs we proceeded in the following manner. First we generated a synthetic cluster assuming that photometric binary stars amount to $30 \%$ of the total sample and that the mass ratio is 1.0 . The photometric errors were switched off. The result is shown in Fig. 1c, where a shift of $0.75 \mathrm{mag}$ in the MS is clearly visible. In the next step we allow the mass ratio to vary in the range of $0.75-1.0$ as this will produce a maximum colour dispersion and the result is shown in Fig. 1d. We find that stars are distributed between the MS envelope of single stars and that of binary stars. Finally we introduced the photometric errors to the CMD shown in Fig. 1d and the final synthetic CMD is shown in Fig. 1e where a broad MS just like the observed CMDs is clearly visible.

In deriving the synthetic CMD, star formation is assumed to be instantaneous. However, there is evidence that indicates non-coeval star formation in a few young open clusters (e.g. Pandey et al. 2001 and references therein). Siess et al. (1997), in the case of the intermediate age cluster Pleiades found that the observed morphology of the CMDs is best reproduced by invoking a large age dispersion $\left(\sim 3 \times 10^{7} \mathrm{yr}\right)$. In such a situation integrated luminosity function (ILF) provides better information in comparison to the LF because a large dispersion in the age does not change significantly the ILF (Chiosi et al. 1989). Therefore, we have compared the observed ILF of the MS stars with those obtained from synthetic CMDs in order to study the effect of photometric binary content on the intrinsic/true MF.

The observational data for three clusters have been taken from the literature and details are given in Table 1. We selected these clusters because the data treatment, e.g, incompleteness correction and removal of field star contamination, was done in a homogeneous way. 
Table 2. Observed luminosity function derived from the $V,(V-$ I) CMD of the clusters (taken from the literature, see Table 1) used in the present study. $N$ denotes field star corrected star counts in $V$-magnitude bins.

\begin{tabular}{|c|c|c|}
\hline Cluster & $\begin{array}{l}\text { Range in } V \\
\text { (mag) }\end{array}$ & $N$ \\
\hline \multirow[t]{4}{*}{ King 5} & $16.0-17.0$ & 25.0 \\
\hline & $17.0-18.0$ & 25.4 \\
\hline & $18.0-19.0$ & 59.0 \\
\hline & $19.0-20.0$ & 28.6 \\
\hline \multirow[t]{4}{*}{ King 7} & $16.0-17.0$ & 26.3 \\
\hline & $17.0-18.0$ & 44.7 \\
\hline & $18.0-19.0$ & 81.7 \\
\hline & $19.0-20.0$ & 81.1 \\
\hline M 37 & $12.0-13.0$ & 56.3 \\
\hline \multirow[t]{7}{*}{ Core } & $13.0-14.0$ & 62.5 \\
\hline & $14.0-15.0$ & 41.4 \\
\hline & $15.0-16.0$ & 37.1 \\
\hline & $16.0-17.0$ & 28.3 \\
\hline & $17.0-18.0$ & 38.9 \\
\hline & $18.0-19.0$ & 27.8 \\
\hline & $19.0-20.0$ & 22.3 \\
\hline M 37 & $12.0-13.0$ & 42.0 \\
\hline Intermediate & $13.0-14.0$ & 79.7 \\
\hline \multirow[t]{6}{*}{ region } & $14.0-15.0$ & 75.4 \\
\hline & $15.0-16.0$ & 102.0 \\
\hline & $16.0-17.0$ & 95.3 \\
\hline & $17.0-18.0$ & 96.0 \\
\hline & $18.0-19.0$ & 71.5 \\
\hline & $19.0-20.0$ & 86.5 \\
\hline M 37 & $12.0-13.0$ & 133.3 \\
\hline \multirow[t]{7}{*}{ Whole } & $13.0-14.0$ & 182.0 \\
\hline & $14.0-15.0$ & 150.3 \\
\hline & $15.0-16.0$ & 203.9 \\
\hline & $16.0-17.0$ & 225.1 \\
\hline & $17.0-18.0$ & 229.8 \\
\hline & $18.0-19.0$ & 156.0 \\
\hline & $19.0-20.0$ & 183.5 \\
\hline
\end{tabular}

It is very important to check the incompleteness of the photometric data which may occur because of various reasons e.g., crowding of stars. The correction for the incompleteness of data is necessary if we want to study the LF/MF of stars in the cluster. The incompleteness of the data used in the present work was estimated using the ADDSTAR routine of the DAOPHOT II. Briefly, the method consists of randomly adding artificial stars (about $10-15 \%$ of the originally detected stars) of known magnitudes and positions into the original frame. The frames were re-reduced using the same procedure used for the original frame. The ratio of the number of stars recovered to those added in each magnitude interval gives the completeness factor (CF) as a function of magnitude. The CF was obtained for the two passbands e.g., $V$ and $I$, and the minimum value of the CF of the pair was adopted to correct the star counts. In most of the cases the sample is complete at the brighter end $(V \sim 16)$ and the CF decreases to 0.7 for stars having $V \sim 20$ (for a detailed discussion see Sagar \& Richtler 1991; Sagar \& Griffiths 1998; Durgapal \& Pandey 2001; Nilakshi \& Sagar 2002).

Another factor that affects the observed luminosity function is the presence of field stars in the cluster region. A statistical criterion was used to remove the field star contamination assuming that the distribution of the field stars is uniform in the vicinity of the cluster. For this purpose an area sufficiently far away ( $\sim 30$ arcmin) from the cluster region was observed. For deriving the LF of a cluster, a $V,(V-I)$ diagram is generally preferred over a $V,(B-V)$ diagram as the former probes about a magnitude deeper than the latter. The LF derived from the $V,(V-I) \mathrm{CMD}$ of the field region was subtracted from the LF of the cluster region. The LFs thus obtained for the clusters used in the study are given in Table 2 (see Durgapal \& Pandey 2001 and Nilakshi \& Sagar 2002 for details). In the case of M 37 the LFs are derived for core (radius $\leq 185^{\prime \prime}$ ), intermediate region $\left(185^{\prime \prime}<\right.$ radius $\left.\leq 480^{\prime \prime}\right)$ and entire cluster region (radius $\leq 1000^{\prime \prime}$ ).

The synthetic CMDs are obtained for various assumed percentage of photometric binary contents, as the binary contents are not available observationally for the clusters used in the study. The MF was varied to match the integrated luminosity function (ILF) of the synthetic CMDs with that of the observed one. The number of stars in the brighter part of the MS is used to fix the proportionality constant of the MF. Figure 2 shows the comparison of the observational ILF of three clusters with their synthetic ILFs for different assumed percentages of photometric binary content along with the values of the MF slope. The results obtained are given in Table 3, which indicates that the value of $x$ for the observed MF (i.e. where the luminosities of the stars were converted without considering the effects of the binary content) is in agreement with the value of the MF slope $x$ obtained for the synthetic CMDs without binary content. Table 3 also indicates that the true/intrinsic value of " $x$ " becomes steeper if the photometric binary fraction is $\geq 40 \%$. This verifies the earlier findings by Sagar \& Richtler (1991) that the derived MF slope becomes significantly steeper if the binary content is higher than $50 \%$.

\section{Estimation of binary content}

The synthetic CMD, where the photometric binary contents are known, can be used to extract information about binary content using the approach described by Durgapal et al. (2001). In brief, to quantify the effect of photometric binary stars on 

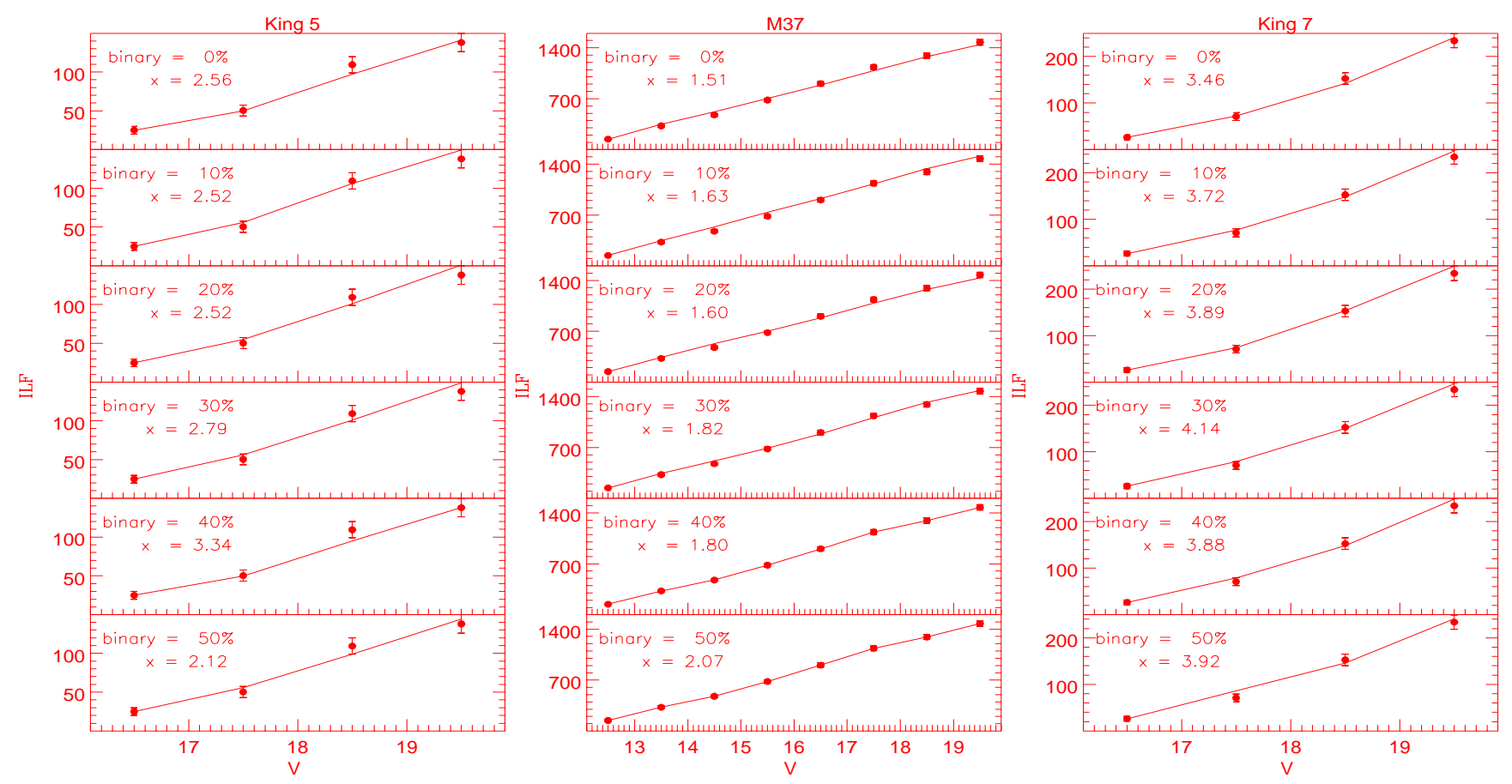

Fig. 2. Synthetic ILFs of clusters King 5, M 37 and King 7 are compared with their observed ILFs. The value of binary content assumed and the values of mass function slopes obtained are also given in the figure. The error bars represents Poisson errors in the observations.

Table 3. The slope of the mass function, $\Gamma=x-1$ obtained by comparing the synthetic ILF for various assumed percentages of binaries with the observed ILF.

\begin{tabular}{|c|c|c|c|c|c|c|c|}
\hline \multirow[t]{2}{*}{ Cluster } & \multirow{2}{*}{$\begin{array}{r}\text { observed } \\
\text { slope }\end{array}$} & \multicolumn{6}{|c|}{ mass function slope for following percent of binary content } \\
\hline & & $0 \%$ & $10 \%$ & $20 \%$ & $30 \%$ & $40 \%$ & $50 \%$ \\
\hline King 5 & $1.69 \pm 0.91$ & $1.56 \pm 0.54$ & $1.52 \pm 0.70$ & $1.52 \pm 0.85$ & $1.80 \pm 1.10$ & $2.34 \pm 0.74$ & $1.12 \pm 0.33$ \\
\hline King 7 & $2.02 \pm 0.24$ & $2.46 \pm 0.44$ & $2.72 \pm 0.63$ & $2.89 \pm 0.61$ & $3.14 \pm 0.48$ & $2.89 \pm 0.54$ & $2.92 \pm 0.58$ \\
\hline M 37 whole & $0.67 \pm 0.12$ & $0.51 \pm 0.11$ & $0.64 \pm 0.08$ & $0.60 \pm 0.08$ & $0.82 \pm 0.10$ & $0.80 \pm 0.11$ & $1.07 \pm 0.10$ \\
\hline M 37 core & $-0.22 \pm 0.15$ & $-0.02 \pm 0.21$ & $-0.22 \pm 0.15$ & $-0.03 \pm 0.18$ & $-0.03 \pm 0.13$ & $-0.41 \pm 0.17$ & $-0.12 \pm 0.10$ \\
\hline M 37 int-region & $0.89 \pm 0.16$ & $0.77 \pm 0.12$ & $0.83 \pm 0.10$ & $1.03 \pm 0.24$ & $1.16 \pm 0.23$ & $1.24 \pm 0.15$ & $1.38 \pm 0.17$ \\
\hline
\end{tabular}

the observed MS of the clusters, we can calculate $\Delta C I=$ $C I_{*}-C I_{\mathrm{MS}}$ at each magnitude, where $C I_{*}$ is either the $(B-V)$ or $(V-I)$ colour of a star and $C I_{\mathrm{MS}}$ is the corresponding colour of the MS. The frequency distribution of $\Delta C I$, summed over all magnitude bins, shows a Gaussian distribution with a peak near $\Delta C I \sim 0.0 \mathrm{mag}$ which is due to the distribution of single stars. The brightening of the MS by 0.75 mag reddens the MS by $\sim 0.15 \mathrm{mag}$ in both $(B-V)$ and $(V-I)$ colour, therefore if photometric binaries are present in the MS sample we can expect another distribution having a peak shifted towards the red side of the MS in the CMDs. The number of stars contributing to this distribution can give an estimate of photometric binary contribution.

Figures $3 \mathrm{a}$ and $3 \mathrm{~b}$ shows the frequency distribution of $\Delta(B-$ $V$ ) for the synthetic CMDs displayed in Figs. 1b and 1e respectively. Figure 3 a shows a Gaussian distribution with a peak at
$\Delta(B-V)=0.0$ mag. Figure $3 \mathrm{~b}$ shows a frequency distribution of $\Delta(B-V)$ for the synthetic CMD with a $30 \%$ photometric binary content and with photometric errors where the curve having its peak at $\Delta(B-V) \sim 0.0$ mag is due to the MS single stars and another curve with a peak at $\Delta(B-V) \sim 0.10 \mathrm{mag}$ is due to the photometric binary content of the MS. The distribution shown by thick lines is the observed composite distribution of the two distributions. The blue edge of the composite distribution is mainly determined by the photometric errors whereas the red edge of the distribution depends on both the photometric binary content and photometric errors.

In principle, the binary fraction can be estimated by comparing either the observed MS $\Delta(B-V)$ or $\Delta(V-I)$ frequency distribution with that obtained for corresponding synthetic CMDs having a binary fraction $0-50 \%$ and the corresponding slope of the mass function as given in Table 3 . 


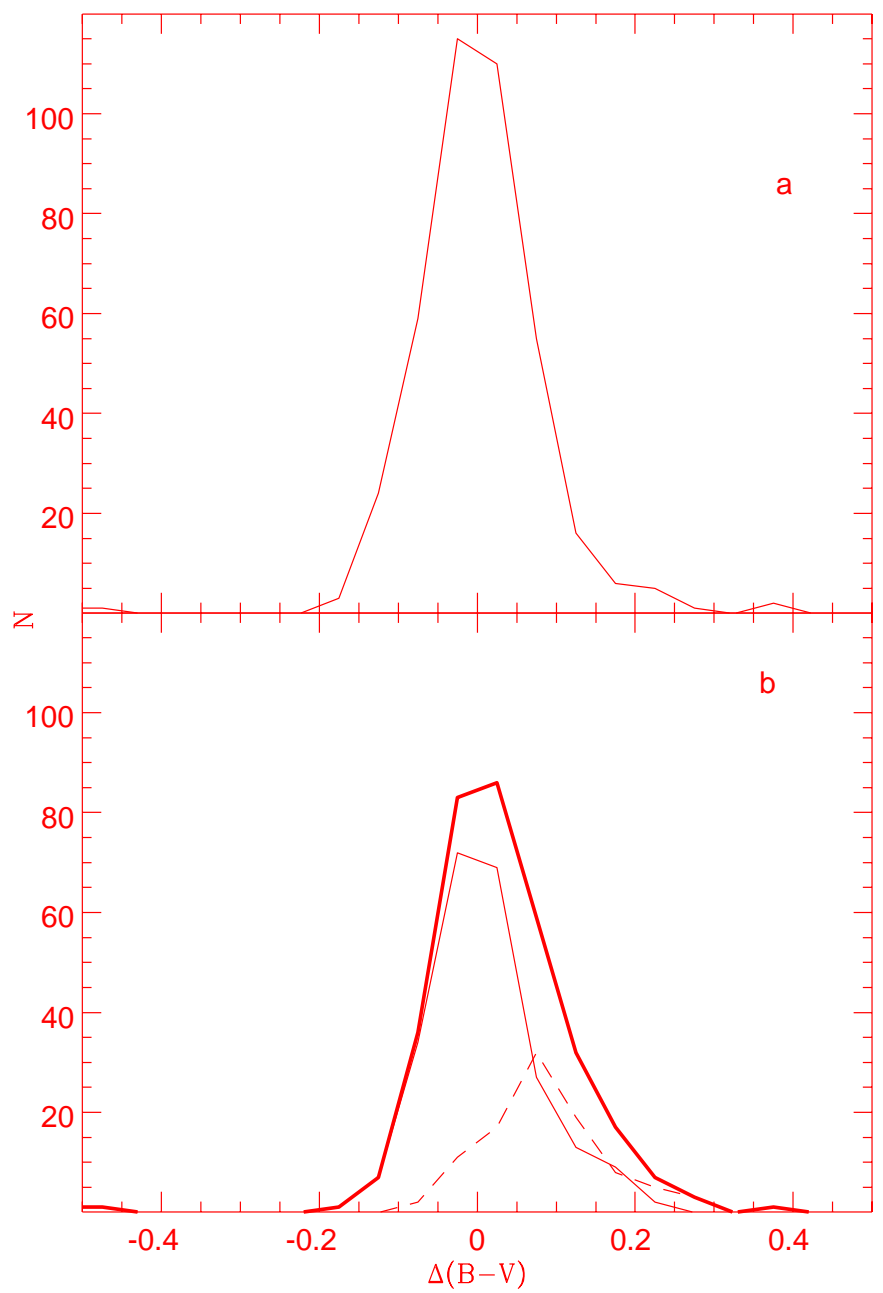

Fig. 3. $\Delta(B-V)$ distribution for synthetic colour-magnitude diagram. The distributions due to single stars and binary stars are shown by thin and dashed curves respectively. The distribution shown by thick lines is the composite distribution of the two distributions.

The $V,(B-V)$ CMD of the three clusters used in the present study shows a better defined ZAMS compared to that in the $V$, $(V-I)$ CMD because the errors in the $I$ band are relatively larger than in the $B$ band. Therefore, we preferred the $V$, $(B-V) \mathrm{CMD}$ to quantify the binaries in these clusters.

Durgapal et al. (2001) have reported a photometric binary content $\sim 10 \%$ and $\sim 20 \%$ for the clusters King 5 and King 7 respectively. Since they estimated the binary content from the well-separated binary distribution peak only, which is located towards the red side of the MS, the binary content reported by them should be considered as the lower limit for the photometric binary content. In Sect. 2 the ILF of synthetic clusters having various photometric binary contents were compared with the observed ILF. In the process we get the value of the MF for different binary contents. To quantify the photometric binary fraction, we compared the observed MS $\Delta(B-V)$ frequency distribution with that obtained for synthetic CMDs having a binary fraction of $0-50 \%$ and a corresponding slope of the mass function as given in Table 3 . To obtain the observed $\Delta(B-V)$ frequency distribution we have to remove the contamination due to field stars present in the cluster region. We removed the contamination statistically using the following procedure. For a randomly selected star in the $(B-V) \mathrm{CMD}$ of the field region, the nearest star in the cluster's $(B-V)$ CMD within $V \pm 0.25$ and $(B-V) \pm 0.125$ of the field star was removed. While removing the stars from the cluster CMD, the number of stars in each luminosity bin was maintained as per the corrected LF given in Table 2 . The MS $\Delta(B-V)$ frequency distribution of this cleaned CMD was compared with the MS $\Delta(B-V)$ frequency distribution of the synthetic CMD.

The comparison of observed and synthetic distributions along with $\chi^{2}$ values for the clusters King 5, King 7 and M 37 is shown in Figs. 4 and 5 respectively. In the case of M 37 we used the sample of two subregions having radius $r \leq 185^{\prime \prime}$ (core) and $185^{\prime \prime}<r \leq 480^{\prime \prime}$ (intermediate region). Figure 5 indicates that the best fit in the inner as well as the intermediate region is obtained for a photometric binary fraction of $30 \%$. Figure 4 gives a photometric binary content of $\sim 30 \%$ and $\sim 20 \%$ for the clusters King 5 and King 7 respectively. A KolmogorovSmirnov test indicates that the expected error in the estimation of binary content is about $10 \%$. Since the cluster M 37 is dynamically relaxed (Nilakshi \& Sagar 2002) a higher binary content is expected in the central region of the cluster. However, in the present study we cannot differentiate variation in the binary content, if it exists in the two regions, in the range of $10 \%-15 \%$ because of the reported uncertainity in estimation of binary content.

Here it is worth mentioning that the mass ratio " $q$ " of the binary content in the present analysis has been varied in a range of $0.75-1.0$ because this range will produce a maximum dispersion in the MS. However, it is well known that binary systems have $q \geq 0.1$. For example, extensive work by Duquennoy \& Mayor (1991) on solar-type field binaries yields that only about $20 \%$ of all binaries have mass ratios $q>0.7$. A similar trend is also found for B-type binaries (Abt et al. 1990). Therefore the analysis using the CMDs presented here probes only a limited fraction of all the binary systems which may be present in the cluster and the binary content reported in the present work can therefore be considered as the lower limit. In the present analysis we also assumed that the binary content does not depend on the mass of the primary because the mass range $\left(0.6 \leq M_{\odot} \leq 2.4\right)$ of member stars of the cluster used in the present work is limited.

\section{Conclusions}

A comparison of the observed CMDs of three open clusters with synthetic CMDs, generated using the theoretical models of Bertelli et al. (1994), gives a photometric binary content of $\sim 30 \%$ in the central region as well as in the outer region of the cluster M 37. On the other hand, in the case of King 5 and King 7, we estimate that $\sim 30 \%$ and $\sim 20 \%$ of the MS stars may be photometric binary stars.

Acknowledgements. We gratefully acknowledge the valuable suggestions given by Dr. G. Duchene which improved the contents of the paper. Thanks are also due to Dr. A. Subramaniam for providing the code which helped in developing the code reported in the present study. 
King 5

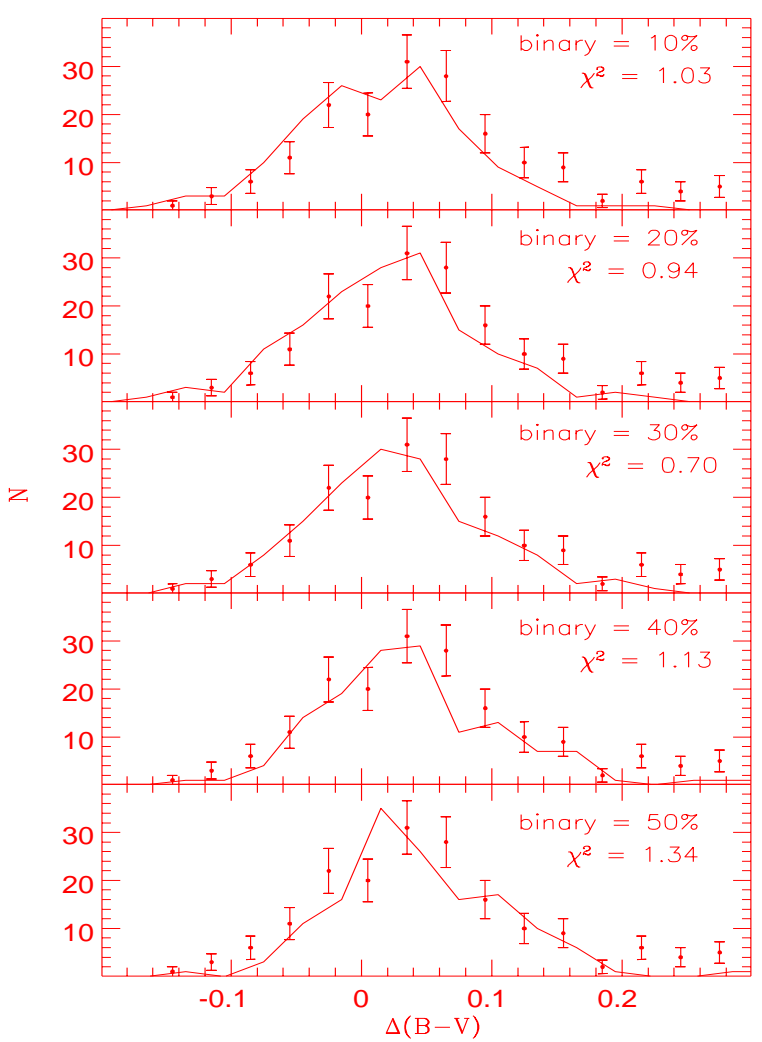

King 7

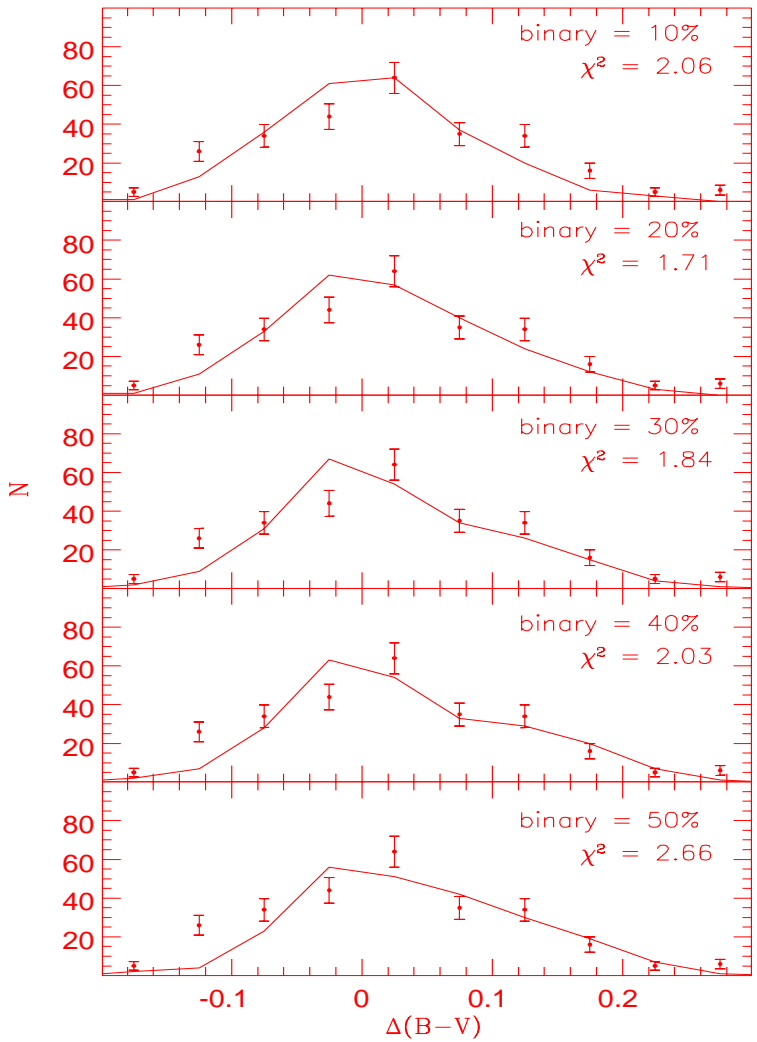

Fig. 4. Observed (filled points with error bars) and synthetic (continuous curve) $\Delta(B-V)$ distribution for King 5 and King 7.

M37 Core

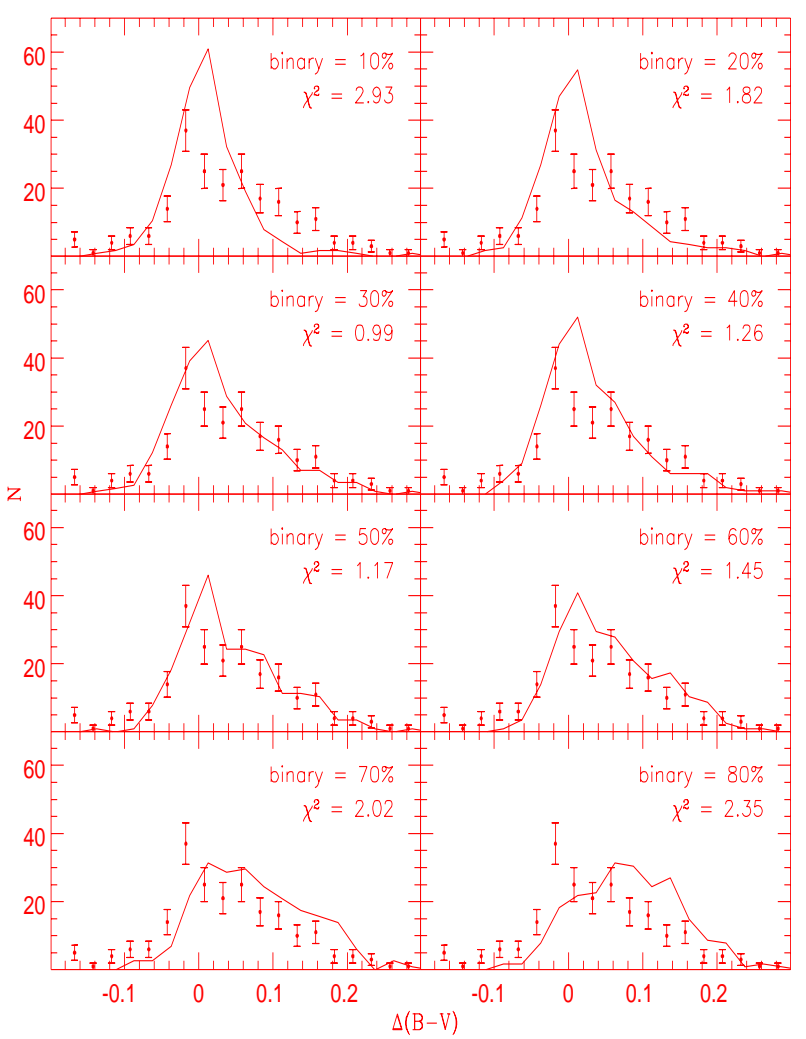

M37 Intermediate region

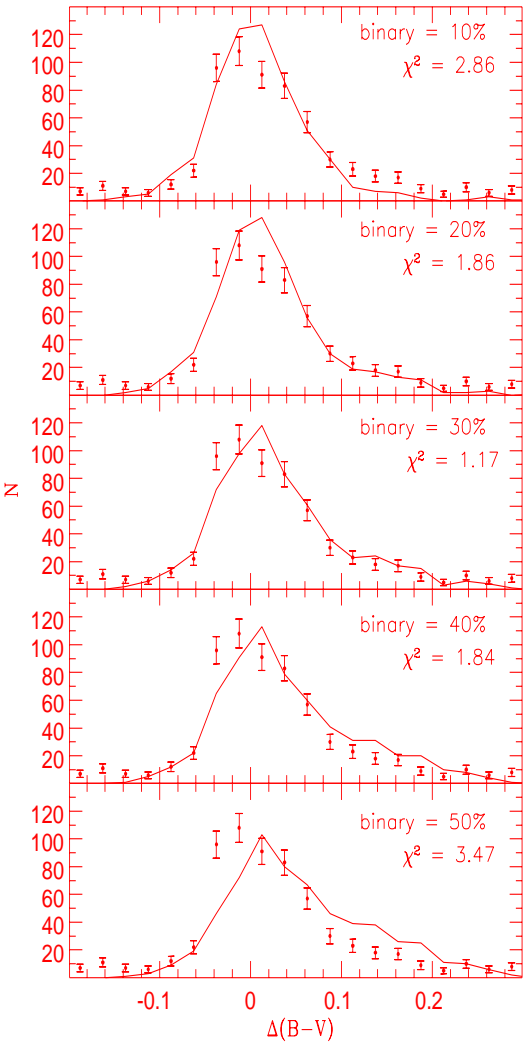

Fig. 5. Same as Fig. 4 but for M 37. 


\section{References}

Abt, H. A., Gomez, A. E., \& Levy, S. G. 1990, ApJS, 74, 551 Aparicio, A., Bertelli, G., Chiosi, C., \& Garcia-Pelayo, J. M. 1990, A\&A, 240, 262

Bertelli, G., Bressan, A., Chiosi, C., Fagitto, F., \& Nasi, E. 1994, A\&A, 106, 275

Brocato, E., Castellani, V., Raimondo, G., \& Romaniello, M. 1999, A\&AS, 136, 65

Bouvier, J., Rigaut, F., \& Nadeau, D. 1997, A\&A, 323, 139

Chiosi, C., Bertelli, G., Meylan, G., \& Ortolani, S. 1989, A\&A, 219, 167

Duquennoy, A., \& Mayor, M. 1991, A\&A, 248, 485

Durgapal, A. K., Pandey, A. K., \& Mohan, V. 2001, A\&A, 372, 71

Durgapal, A. K., \& Pandey, A. K. 2001, A\&A, 375, 840
Jeffries, R. D., Thurston, M. R., \& Hambly, N. C. 2001, A\&A, 375, 863

Kaluzny, J., \& Rucinski, S. M. 1995, A\&AS, 114, 1

Mermilliod, J.-C., \& Mayor, M. 1989, A\&A, 219, 125

Nilakshi \& Sagar, R. 2002, A\&A, 381, 65

Patience, J., Ghez, A. M., Reid, I. N., Weinberger, A. J., \& Matthews, K. 1998, AJ, 115, 1972

Pandey, A. K., Mahra, H. S., \& Sagar, R. 1990, AJ, 99, 617

Pandey, A. K., Nilakshi, Ogura, K., Sagar, R., \& Tarusawa, K. 2001, A\&A, 374, 504

Sagar, R., \& Richtler, T. 1991, A\&A, 250, 324

Salpeter, E. E. 1955, ApJ, 121, 161

Siess, L., Forestini, M., \& Dougados, C. 1997, A\&A, 324, 556

Subramaniam, A., \& Sagar, R. 1995, A\&A, 297, 695

Yadav, R. K. S., \& Sagar, R. 2001, MNRAS, 328, 370 